\title{
Modular Terpene Synthesis Enabled by Mild Electrocatalytic Couplings
}

Authors: Stephen J. Harwood ${ }^{1 \dagger}$, Maximilian D. Palkowitz ${ }^{1 \dagger}$, Cara N. Gannett ${ }^{2}$, Paulo Perez ${ }^{3}$, Zhen $\mathrm{Yao}^{4}$, Lijie Sun ${ }^{4}$, Hector D. Abruña ${ }^{2 *}$, Scott L. Anderson ${ }^{3 *}$, and Phil S. Baran ${ }^{1 *}$

\author{
Affiliations: \\ ${ }^{1}$ Department of Chemistry, Scripps Research, La Jolla, CA, 92037, USA. \\ ${ }^{2}$ Department of Chemistry and Chemical Biology, Baker Laboratory, Cornell University, Ithaca, \\ NY, 14853, USA \\ ${ }^{3}$ Department of Chemistry, University of Utah, 315 S. 1400 E., Salt Lake City, UT, 84112, USA \\ ${ }^{4}$ Asymchem Life Sciences (Tianjin) Co., Ltd. No. 71, $7^{\text {th }}$ Ave., TEDA Tianjin, 300457, P.R. \\ China \\ ${ }^{\dagger}$ These authors contributed equally to this work. \\ *Correspondence to: hda1@ cornell.edu, anderson@chem.utah.edu, pbaran@scripps.edu
}

\begin{abstract}
The synthesis of terpenes is a large field of research that is woven deeply into the history of chemistry. Terpene biosynthesis is a case-study of how the logic of a modular design can lead to diverse structures with unparalleled efficiency. This work mimics Nature by leveraging modern Ni-catalyzed electrochemical $\mathrm{sp}^{2}-\mathrm{sp}^{3}$ decarboxylative coupling reactions-enabled by Ag-nanoparticle modified electrodes - to intuitively assemble terpene natural products and complex polyenes. The step-change in efficiency of this approach is exemplified through the scalable preparation of 13 complex terpenes, which minimized protecting group manipulations, functional group interconversions, and redox fluctuations. Finally, the mechanistic aspects of the essential functionalized electrodes are studied in depth through a variety of spectroscopic and analytical techniques.
\end{abstract}

Main Text: The study of terpene synthesis holds a special place in the annals of organic synthesis, with the formalization of the stereoelectronic effect, conformational analysis, rules for pericyclic reactions, and even retrosynthetic analysis stemming from this field. ${ }^{1}$ Beyond synthesis, the pivotal role that terpenes play in nature and medicine has inspired practitioners from a wide spectrum of the scientific community. ${ }^{2,3}$ As medicines, they exhibit broad activity ranging from modulation of human physiology (e.g., steroid hormones, cannabinoids) to amelioration of disease (e.g., Taxol®, artemisinin). ${ }^{2}$ Terpenes also perfuse the fine chemicals industry and are found in commercial fragrances and food additives. ${ }^{4}$ Not surprisingly, this class of natural isolates has inspired numerous total syntheses, a legendary example is Johnson's landmark synthesis of progesterone in 1971 (Figure 1A). ${ }^{5}$ As one of the first biomimetic terpene syntheses, it validated the Stork-Eschenmoser hypothesis by stitching together the polycyclic steroid core through a bold cation-olefin cyclization. ${ }^{6}$ This tactic subsequently shaped the landscape of future chemical approaches to such molecules through its ability to generate polycyclic ring-systems and multiple stereocenters from prochiral alkenes. Polyene cyclization is still an active area of research in the modern era, as evidenced by the steady development of new polycyclization reactions and 
enantioselective variants. ${ }^{7}$ Although the power of cation-olefin cyclization is undisputed, the construction of polyunsaturated precursors remains oddly challenging. Retrosynthetic strategies to forge such entities are still plagued with a non-intuitive logic where building blocks used do not clearly map onto the product into which they are ultimately incorporated. Specifically, these approaches are non-modular and individually target each poly-olefin synthesized. Furthermore, they often lack complete control of olefin geometry (a critical feature controlling the resulting $\mathrm{sp}^{3}$ stereochemistry), and require multiple functional group interconversions (FGIs). ${ }^{8}$ The current barrier to rapid, modular, and controlled polyene construction therefore limits the effectiveness of what is arguably one of the most powerful complexity-inducing chemical transformations known. Meanwhile, steady advancement in the development of methodology for creating $\mathrm{sp}^{2}-\mathrm{sp}^{3}$ linkages has pointed towards a simpler approach to polyene synthesis (Figure 1B), ${ }^{9,10}$ The cyclase phase of natural terpene assembly points to inherent advantages of a modular approach as a simple building block like isopentenyl pyrophosphate (IPP) can be intuitively mapped onto the final polyene product. ${ }^{11}$ The goal of this study was to mimic this strategy for modular terpene synthesis by focusing on disconnecting $\mathrm{sp}^{2}-\mathrm{sp}^{3}$ bonds directly to arrive at simple carboxylic acid precursors (Figure 1C). By combining the learnings of decarboxylative $\mathrm{C}-\mathrm{C}$ bond formation and electrochemical cross-electrophile coupling with a new application of in situ electrode functionalization we show how halo-acid modules can be iteratively coupled resulting in more logical retrosynthetic analyses that reduce step-counts, reliance on pyrophoric reagents, and remove non-ideal manipulations. ${ }^{12,13}$ The scalable access to 13 terpene natural products exemplifies the strategic power of this logic. Furthermore, the mechanistic interplay between the

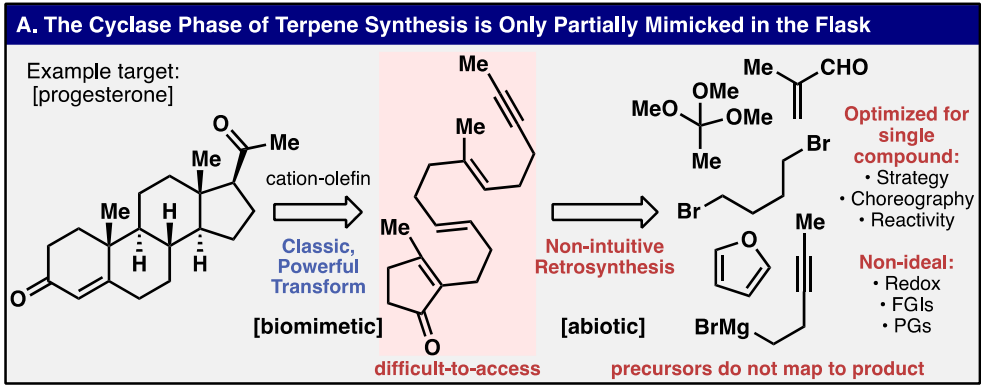

B. Modular Approaches to Polyunsaturated Molecules in the Flask and in Nature
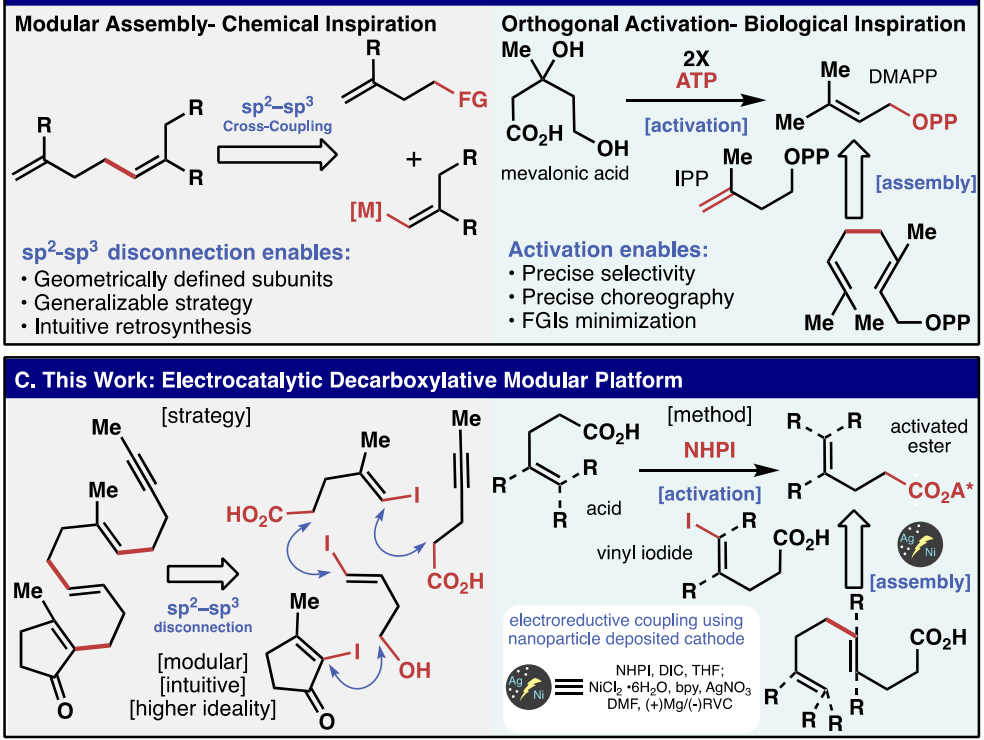

Fig. 1. The synthesis of polyene cyclization precursors remains a challenge to chemists. Taking inspiration from chemical and natural precedent, this work developed a modular platform constructing $\mathrm{sp}^{2}-\mathrm{sp}^{3}$ bonds utilizing an electrochemical reaction and functionalized electrodes. (A) Retrosynthetic analysis of Johnson's classic total synthesis of progesterone. (B) The advantages of a prototypical $\mathrm{sp}^{2}-\mathrm{sp}^{3}$ disconnection and the biosynthesis of geraniol pyrophosphate. Adenosine triphosphate (ATP) activation of an alcohol generates the reactive chemical feedstocks necessary for terpene synthesis. (C) Combining the advantages of the $\mathrm{sp}^{2}-\mathrm{sp}^{3}$ disconnection and nature's modular and selective activation strategy required development of a mild reaction using nickel electrocatalysis and $\mathrm{Ag}$-nanoparticle functionalized electrodes. 
Ag-embedded heterogeneous interface and the homogenous Ni-catalyst exemplifies the untapped potential of functionalized electrodes in synthetic organic electrochemistry.

The execution of the plan outlined above would require a departure from many of the (alkenyl)$\mathrm{sp}^{2}-\mathrm{sp}^{3}$ disconnection strategies previously utilized that combine alkyl organometallic reagents or bornates with vinyl halides (Figure 1B). ${ }^{9,10}$ The functional group compatibility needed (tolerating free carboxylic acids, for example) and a desire to minimize functional group interconversions (e.g. $\mathrm{R}-\mathrm{X}$ to $\mathrm{R}-\mathrm{BR}_{2}$ ) was vividly illustrated in our initial forays (Figure 2). Here we enlisted the recently disclosed decarboxylative alkenylation with organozincs (prepared through lithium halogen exchange). ${ }^{14}$ In the coupling with redox-active ester (RAE) 2, more than three equivalents of $\mathbf{1}$ and six equivalents of $t$ - $\mathrm{BuLi}$ under cryogenic conditions were required to generate the organometallic. Deprotection and oxidation of the resulting product $\mathbf{3}$ set the stage for the ensuing coupling. Although conceptually attractive, this approach fell short of the modular and mild aspirations of the initial plan (see SI for discussion). The seminal work of Périchon and Nédélec on electrochemical reductive crosscoupling was inspirational as it demonstrated the coupling of vinyl halides with electronically biased alkyl halides in a simple undivided cell. ${ }^{15}$ Additionally, reports adapting this reactivity to aryl-alkyl cross-coupling using alkyl RAEs as alternatives to

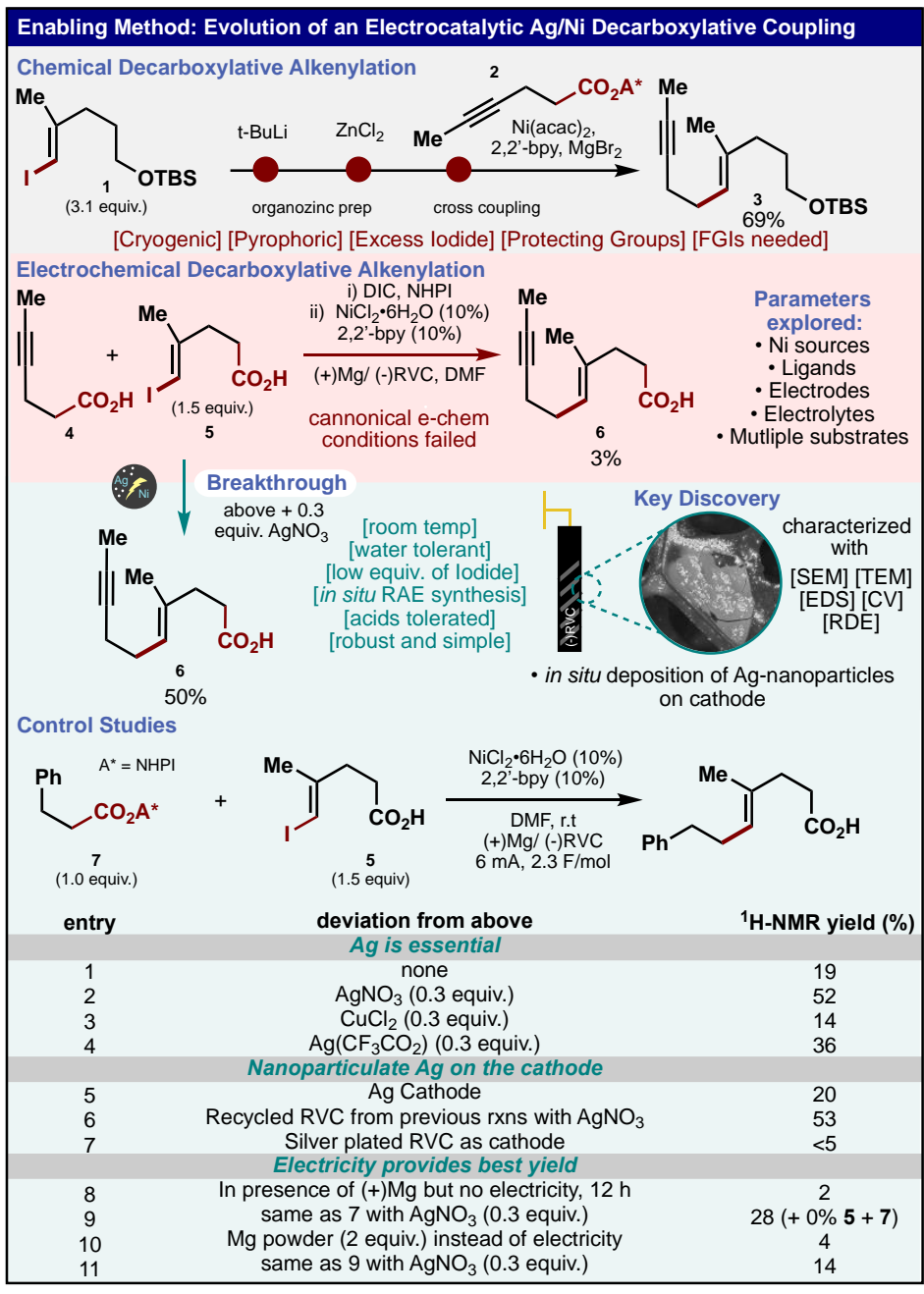

Fig. 2. The discovery and optimization of the electrocatalytic methodology is described. The published decarboxylative alkenylation is a prototypical example of literature methods to construct $\mathrm{sp}^{2}-\mathrm{sp}^{3}$ bonds requiring harsh reagents, cryogenic temperatures, protecting groups and functional group interconversions (FGIs). Initial electrochemical conditions were insufficient to accomplish the envisioned plan to crosscouple in situ activated acids and halo-acid modules. The addition of $\mathrm{AgNO}_{3}$ (0.3 equiv.) to the reaction was a breakthrough. Control experimentation suggests that nanosilver on the cathode is responsible for the improved yield and that electrochemistry offers a unique advantage over other systems. alkyl halides have emerged. ${ }^{16}$ Inspired by these precedents, initial conditions were developed that enabled a more functional group tolerant reductive coupling of pre-generated RAEs with vinyl iodides. Unfortunately, after initial optimization efforts (see SI Tables S31-36) the use of free acid 5 in this milder coupling only provided trace quantities of $\mathbf{6}$ using an in situ protocol for the conversion of $\mathbf{4}$ to the corresponding RAE. An extensive series of optimization experimentation 
was performed in hope of overcoming this apparently intractable problem. Many of the traditional experimental variables associated with the optimization of electrochemical reactions such as electrolyte and electrode were re-evaluated to no avail. Modification of the properties of the Nibased electrocatalytic system were also re-explored by screening ligands, Ni-sources, and other metals known to promote reductive coupling. Finally, given the heterogeneous nature of electrochemistry, efforts to modify the surface of the electrode were pursued. Two metals that are known to readily and reproducibly plate onto carbon electrodes, $\mathrm{Cu}$ and $\mathrm{Ag}$, were explored with the latter providing a stunning increase in reaction efficiency and functional group compatibility. ${ }^{17}$ Thus, addition of 0.30 equiv. $\mathrm{AgNO}_{3}$ to the standard reductive coupling conditions $\left[\mathrm{NiCl}_{2} \bullet 6 \mathrm{H}_{2} \mathrm{O}\right.$ (10 mol\%), 2,2'-bpy (10 mol\%), DMF, r.t] resulted in 50\% isolated yield of 6 . The precise nature of this electrode modification was studied in detail (vide infra) and supported the notion that Agbased nanoparticles were present and responsible, at least in part, for this enhanced outcome. Before applying these newly developed conditions, a number of control studies were undertaken as illustrated at the bottom of Figure 2. Using coupling partners 5 and $\mathbf{7}$ as model substrates that give measurable yields without $\mathrm{AgNO}_{3}$, several experiments confirmed that the $\mathrm{Ag}$ ion was the essential additive (entries 1-3). Entries 4-6 corroborate the role of Ag-based nanoparticles as the use of pure Ag electrodes or Ag-plated electrodes did not work well whereas Ag-nanoparticles, deposited on RVC electrodes, could be recycled without the need for added Ag. Finally, the critical role of electrochemistry in this process was confirmed (entries 7-10) by running the reaction in the absence of current or by employing stoichiometric chemical $(\mathrm{Mg})$ reductants; although, addition of $\mathrm{Ag}$ to those non-electrochemical processes did lead to notable improvements. In the final optimized manifestation, a free carboxylic acid (1.0 equiv.) could be converted to the NHPI-RAE (DIC, NHPI, 1-3 h) followed by direct addition of the reaction medium containing vinyl iodide (1.5 equiv.) and $\mathrm{Ni}$ catalyst in DMF $(0.07-0.25 \mathrm{M})$; the solution was then added to a commercial electrochemical cell fit with a sacrificial Mg-anode and RVC-cathode containing $\mathrm{AgNO}_{3}(0.3$ equiv.). Then, the reaction was electrolyzed (ca. $2.5 \mathrm{~F} / \mathrm{mol}, 2.5 \mathrm{~h}$ ) at ambient temperature.

With a viable method in hand for chemoselective and modular $\mathrm{C}-\mathrm{C}$ coupling, execution of the blueprint outlined in Figure 1 could be explored. Rather than pursue a tabular listing of coupling partners to illustrate functional group tolerance and geometric control (all stereoretentive), the value of the methodology was exemplified through the total or formal synthesis of 13 terpene natural products (Figure 3). A range of functional groups is tolerated, including epoxides, alkynes, alcohols, free carboxylic acids, esters, ethers, ketones, enones, aldehydes, electron-rich (hetero)aromatics, $\beta$-keto esters, and skipped dienes. The simplification enabled by this method is apparent in three poly-olefin precursors prepared en route to complex terpene natural products: progesterone (8), celastrol (9), and isosteviol (10). A protecting group free synthesis of progesterone precursor 8 begins with the electrochemical cross-coupling of two simple acids 4 (in situ activated) and $\mathbf{5}$ (prepared in two steps via carboiodination and oxidation). The product acid $\mathbf{6}$ was next in situ activated and coupled to a vinyl iodide bearing a free alcohol (11). Alcohol 12 was converted to the bromide (13) via Appel conditions and coupled to 2-iodo-3methylcyclopentenone using an electrochemical reaction inspired by Hansen's electrochemical conditions (See SI Tables S3-6) to deliver the desired polyene endpoint (8) directly. ${ }^{18}$ In contrast, similar reported couplings have employed Suzuki conditions which required three steps to prepare a suitable trifluoroborate-containing coupling partners. ${ }^{19}$ This modular and intuitive approach takes place in six steps (longest linear sequence, LLS) relying on a single FGI and oxidation step without resorting to any protecting groups. In contrast, Johnson's synthesis of progesterone arrived at the target polyene in 8 steps (LLS) with two FGI's and two PG-related steps. ${ }^{5}$ In 2016, 
Dudley and coworkers published a 10-step total synthesis of progesterone which also prepares polyene 8 in six steps LLS with one FGI and one oxidation. ${ }^{19}$

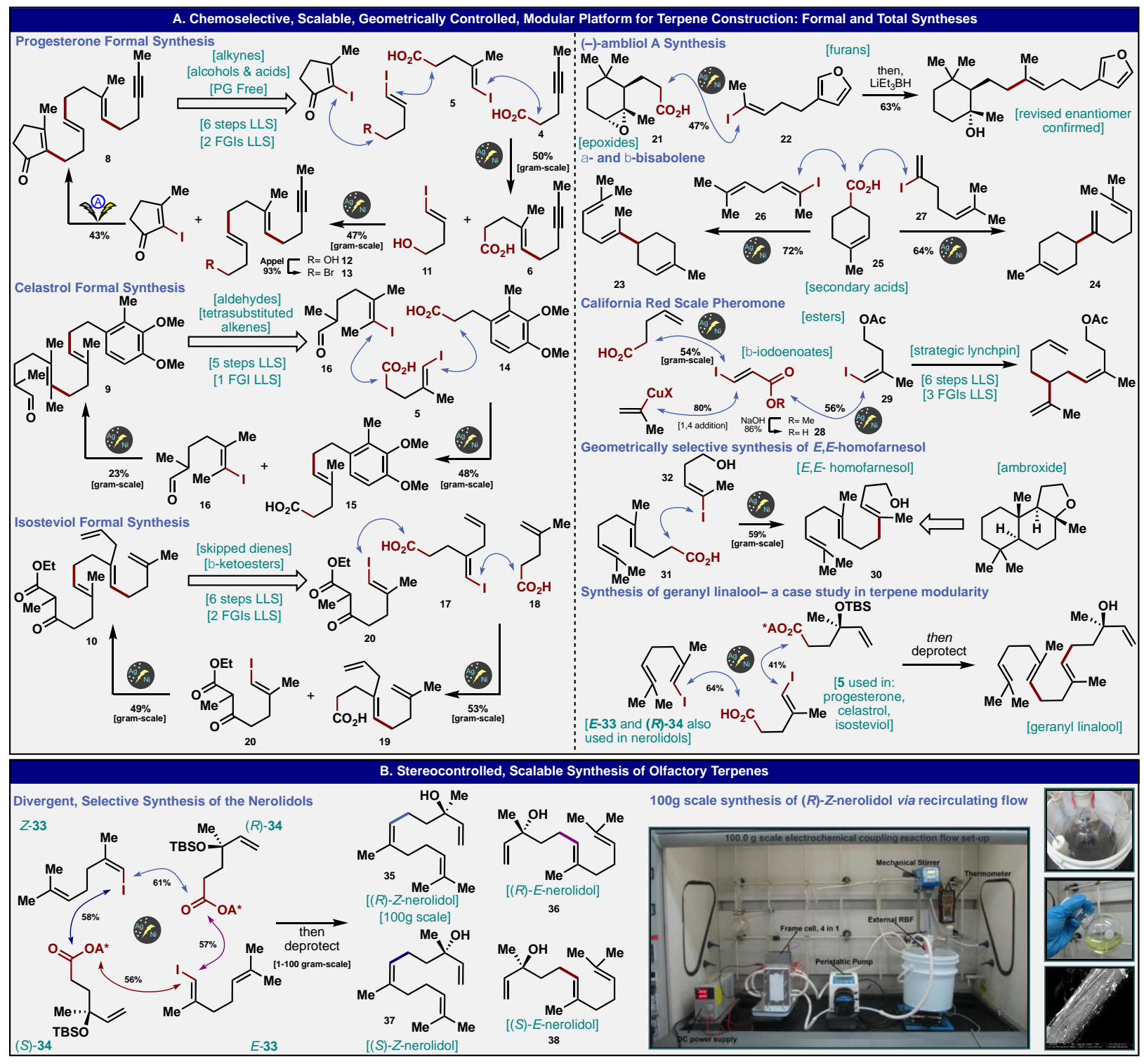

Fig. 3. The utility and scalability of the developed electrocatalytic methodology is showcased through the formal or total synthesis of 13 natural products. (A) Three polyene cyclization precursors and five natural products are synthesized through iterative electrochemical cross-electrophile couplings showing a functional group tolerance inclusive of alkynes, alcohols, acids, esters, ketones, enones, aldehydes, ethers, epoxides, electron-rich heteroaromatics and skipped dienes. (B) A unified and scalable synthesis of the four nerolidol isomers is described. A recirculating flow system was used to conduct a $100 \mathrm{~g}$-scale electrochemical crosscoupling. The large image showcases the complete flow system consisting of a frame cell, power source, peristaltic pump, round bottom flask reservoir, and a mechanical stirrer. On right: (top) reaction reservoir, (middle) pure product after column chromatography, (bottom) SEM image of the Ag-nanoparticle embedded carbon felt cathode post-reaction.

Siegel's elegant approach to 9 leveraged a unique polyene cyclization precursor bearing a tetrasubstituted alkene and an electron rich arene. ${ }^{20}$ Cleverly, inherent symmetry was exploited to arrive at the polyene in 9 steps, using one FGI and one oxidation. The use of an HWE reaction led to a 
6:1 mixture of $E / Z$ isomers, highlighting the challenges associated with approaches that construct polyolefins through Wittig-like transforms. In contrast, the modular assembly approach breaks bonds adjacent to the olefins and programs the alkene geometry at the outset. The synthesis commences with in situ activation of acid $\mathbf{1 4}$ and electrochemical coupling with the same vinyl iodide $\mathbf{5}$ used in the synthesis of $\mathbf{8}$. The product acid $\mathbf{1 5}$ was subsequently activated and coupled with tetra-substituted vinyl iodide $\mathbf{1 6}$ bearing a reactive aldehyde to complete the formal synthesis endpoint of celastrol (9) in 5 steps.

Snider's impressive synthesis of isosteviol relied on their pioneering oxidative radical cascade methodology from polyene 10-prepared in 10-steps (LLS) with 2 FGI's and 3 redox manipulations. $^{21}$ Our initial attempts using more traditional organometallic cross-coupling chemistry (Figure 2) yielded no success in the first coupling (see SI, Figure S9). Using vinyl organolithium reagents led to a retro-lithium ene to expel allyl lithium and treating the allyl vinyl iodide 17 with organometallic reagents led to recovery of vinyl iodide. The only conditions identified capable of forging the desired bond between the acid $\mathbf{1 8}$ and skipped diene $\mathbf{1 7}$ were the developed electrochemical conditions, which gratifyingly gave the desired product acid 19 in 53\% isolated yield on gram-scale. The resulting acid 19 was activated and coupled to vinyl iodide 20 bearing the reactive $\beta$-ketoester functionality (a testament to the functional group compatibility of the developed conditions) providing more than a gram of polyene $\mathbf{1 0}$ in 6 steps LLS.

To further test the capabilities of the developed methodology, a broad range of terpenes was targeted. The diterpene, ambliol A, first isolated from the marine sponge Dysidea amblia by Faulkner in 1981 off of the Pacific coast (La Jolla) was chosen as an attractive target to synthesize for its antibiotic activity and unique functionality. ${ }^{22,23}$ Only recently (2015) was an enantioselective synthesis of (+)-ambliol A accomplished by Serra et al. utilizing an enzymatic resolution in 16 steps LLS. ${ }^{24}$ That synthesis led to a revision of the original enantiomeric assignment. In contrast, a convergent synthesis of (-)-ambliol A utilizing the developed electrochemical conditions was achieved in just five steps LLS by coupling the enantiopure epoxy-acid 21 (confirmed via X-ray, See SI, Figure S110) with furan 22 in $47 \%$ yield followed by reductive epoxide opening ( $\left.\mathrm{LiEt}_{3} \mathrm{BH}\right)$ thus unambiguously confirming its stereochemical revision and enabling the first synthetic access to the natural enantiomer.

The sesquiterpenes $(E)-\alpha$ - and $\beta$-bisabolenes (23 and $\mathbf{2 4}$ ), which function in nature as pheromone molecules for a number of insects, were an opportunity to test the electrochemical cross-coupling on secondary acids. ${ }^{25}$ Targeting the central $\mathrm{sp}^{2}-\mathrm{sp}^{3}$ junction for disconnection, syntheses of $\mathbf{2 3}$ and $\mathbf{2 4}$ diverged from secondary acid 25 and enlisted vinyl iodide $\mathbf{2 6}$ or 27 to furnish 23 and 24 in $72 \%$ and $64 \%$ yield, respectively. The more stabilized secondary radical generated from the acid behaved admirably under the standard reaction conditions providing access to both natural products in three steps LLS.

The California red scale sex pheromone, produced by one of the most significant citrus pests, was originally identified in 1977 by Gieselmann and co-workers and is used by the females to attract males. ${ }^{26}$ Its synthesis has applications to the agricultural industry as a replacement for virgin female traps in monitoring the pest's population. Strategically, a synthesis was envisioned that leveraged a $\beta$-iodo-enoate as a lynchpin fragment capable of forging $3 \mathrm{key} \mathrm{C}-\mathrm{C}$ bonds off the central two carbon linker. This concept was realized by first cross-coupling 4-pentenoic acid with methyl $(E)$-3-iodoacrylate in 54\% yield, followed by a conjugate addition and saponification in $80 \%$ and $86 \%$ respectively to provide acid $\mathbf{2 8}$. The synthesis could be completed by coupling 28 and acetate 29, however, upon generation of the primary radical under the reaction conditions, it 
would undergo a 5-exo-trig cyclization before cross-coupling of the vinyl iodide could take place. This problem was obviated by increasing the catalyst loading to $40 \mathrm{~mol} \%$ to provide California red scale pheromone in $56 \%$ yield and six steps LLS. ${ }^{27}$

The importance of the homologated terpene $(E, E)$-homofarnesol $(\mathbf{3 0})$ stems from its use as a cyclization precursor to the vital terpene Ambrox $₫$ (ambroxide) - a molecule with a storied history dating back to the $15^{\text {th }}$ century. ${ }^{28}$ Many of the reported syntheses of $\mathbf{3 0}$ rely on [2,3] rearrangements of the nerolidols that lead to mixtures of $E / Z$ isomers which, if not carefully separated, give complex mixtures of cyclization products that harm its fragrant properties. ${ }^{29}$ In contrast, acid 31 (readily prepared from geranyl bromide and diethyl malonate) was in situ activated and coupled with vinyl iodide $\mathbf{3 2}$ bearing a free alcohol to arrive directly at $(E, E)$ homofarnesol (>4 grams prepared in single pass).

The diterpene geranyl linalool was synthesized through two sequential cross-couplings of common fragments: one of which was employed in the synthesis of progesterone and celastrol and the other two of which were utilized to access nerolidols (Figure 3B). This mix-and-match strategy for opportunistically accessing naturally occurring terpenes highlights the advantage of using the modular logic also employed in Nature's cyclase phase.

Notwithstanding the value of the disclosed methods to the academic pursuit of complex terpene total synthesis, nowhere is the study and utilization of linear terpenes more apparent than in the fragrance and flavor industry (>\$30-billion-dollar per annum). ${ }^{4}$ Given the sensitivity of human olfactory receptors, single terpene isomers of high purity are desired to ensure precise flavor and fragrance profiles because small changes in structure can create very different properties. ${ }^{30,31}$ One class of terpene targets, nerolidol, seemed particularly relevant in this context. A unified, controllable synthesis of the linear terpenes $(E)$ - and $(Z)$-nerolidol, produced naturally as a mixture of four isomers derived from nerol and geraniol, has remained an unanswered synthetic challenge for over four decades. Whereas their synthesis and separation have been explored, preparative methods of separation or synthesis are expensive and laborious. ${ }^{32}$ Indeed, the extreme price disparity between the racemic mixture of isomers (ca. \$0.09/gram SigmaAldrich), and the geometrically pure racemate (trans: \$610/g, Sigma-Aldrich; cis: \$3432460/gram, various suppliers) is cost-prohibitive while the enantiopure natural products are not commercially available. Despite the difficulty in purification, nerolidol is estimated to be used per annum in quantities between 10 to 100 metric tons and appears in products like shampoos, perfumes, detergents and as a flavor additive. The need for a selective and programmable synthesis of these four isomers stems from their differing fragrance profiles.

To synthesize $(E)$ - and $(Z)$ - nerolidols, we imagined these isomers could arise from two geometrically differentiated vinyl iodides $(\boldsymbol{E}-\mathbf{3 3}$ and $\boldsymbol{Z}-\mathbf{3 3}$, respectively) and enantiomeric RAEs derived from $(R)$ - and $(S)$-linalool, respectively $(\boldsymbol{R}-\mathbf{3 4}, \boldsymbol{S}-\mathbf{3 4})$. A simple mix-and-match union of the modules resulted in the controlled synthesis of 35-38 in 44\%-60\% yield after deprotection. As a proof-of-concept for the scalability of the method, 35 was arbitrarily chosen and the electrochemical coupling was performed on 100g scale (at Asymchem, see inset photos). Of note, analysis of the electrodes in this large-scale flow run confirmed that Ag-nanoparticles were present at a surface coverage of $\mathrm{Ag}$ analogous to small scale reactions requiring only 0.07 equiv. of $\mathrm{Ag}$ (see SI, Figures S47-48). 


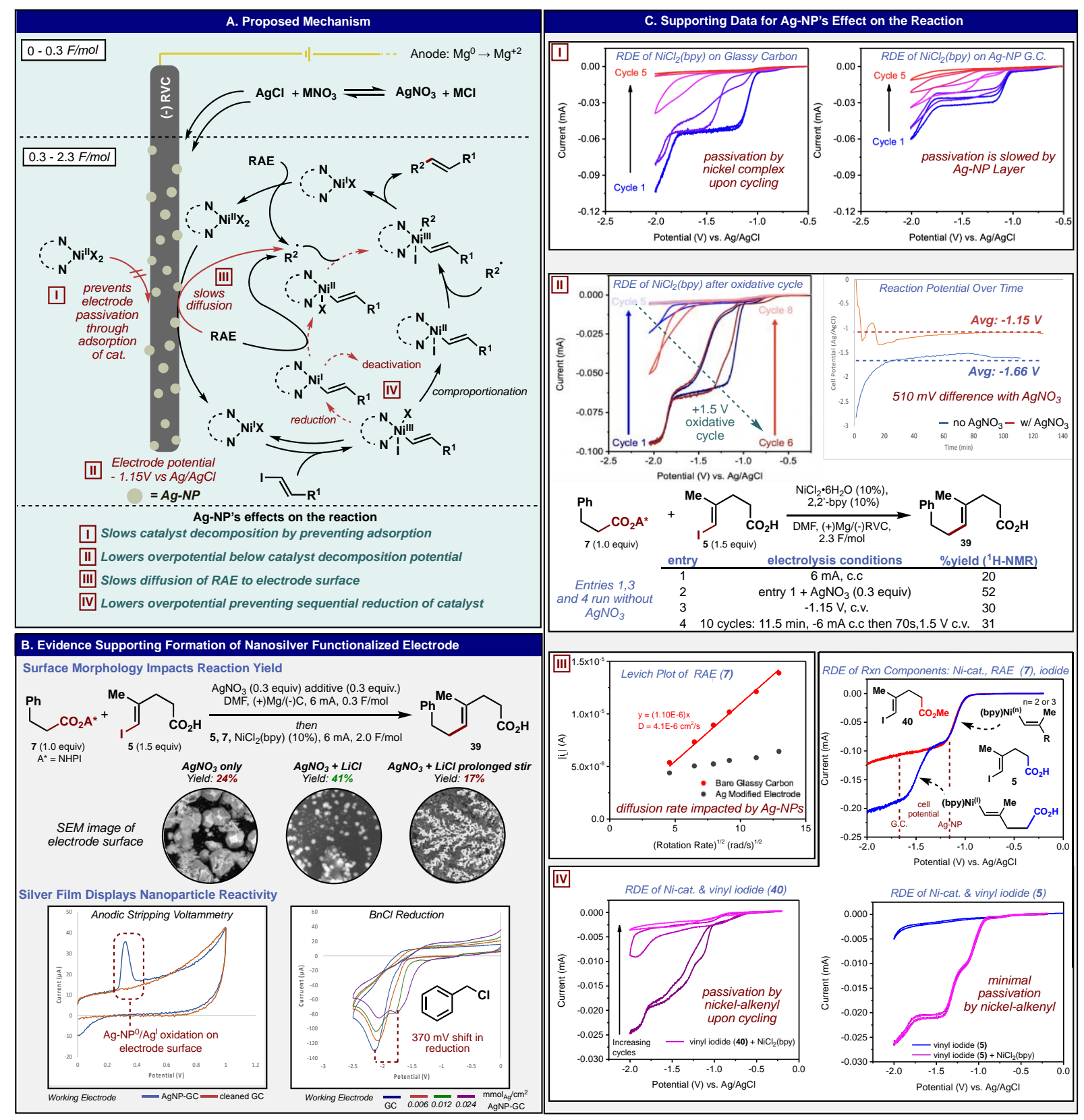

Fig. 4. The existence of silver nanoparticles on the electrode surface is confirmed and the specific roles of the silver nanoparticle layer are outlined. (A). A mechanism is proposed with roman numerals indicating points of interaction with the Ag-nanoparticle layer. The silver layer slows catalyst decomposition (I), decreases overpotential so that the electrode potential falls below the decomposition potential (II), limits diffusion and mass transport of the RAE to the electrode surface (III), and adsorbs the acid-bearing vinyl iodide to the electrode surface (IV). (B) Control studies and S/TEM imaging identify that the reaction conditions produce Ag-nanoparticles on the electrode surface, a halide source is required and photodecomposition of the silverhalide solution before electrolysis prevents nanoparticulate formation and diminishes the reaction yield. (C) Rotating disk electrode (RDE) voltammetry provides supporting evidence for the differences between the Agnanoparticle functionalized and unfunctionalized glassy carbon electrodes. [I] RDE voltammetric profiles (at $1600 \mathrm{rpm}$ ) of the Ni(bpy) catalyst at bare and Ag-NP modified glassy carbon electrodes. [II] Regeneration of activity, time evolution of cell potential and product yields under various electrolysis conditions. [III] Levich plots (I vs $\omega^{-1 / 2}$ ) for $\mathrm{NiCl}_{2}$ (bpy) catalyst and RAE 7. [IV] RDE voltammetric profiles of vinyl iodides 5 (right) and 40 (left) at bare glassy carbon electrodes exhibiting $\mathrm{EC}_{\mathrm{cat}}$ kinetics (note the two reduction waves). $\mathrm{RDE}$ voltammetric profile of reaction components (top) ( $1 \mathrm{mM} \mathrm{NiCl} 2$ (bpy), $10 \mathrm{mM} \mathrm{RAE} \mathrm{7,15} \mathrm{mM} \mathrm{vinyl} \mathrm{iodide} \mathrm{(40,}$ red trace) (5, blue trace). Second reduction wave at present $-1.48 \mathrm{~V} \mathrm{vs} \mathrm{Ag} / \mathrm{AgCl}$ with 5, but not with $\mathbf{4 0}$. 
While the observations from the above syntheses suggested that the homogenous Nicatalysis behaved according to literature precedent for radical cross-coupling ${ }^{27}$, the impact of electrode surface functionalization on this methodology warranted further study (Figure 4). An induction period was observed that corresponded in duration to the amount of $\mathrm{AgNO}_{3}$ added suggesting that silver reduction preceded the cross-coupling reaction (see SI, Figure S19-20). Despite nano-silver's use in catalysis, to the best of our knowledge, the use of silver nanoparticle functionalized electrodes has not been proposed to support and/or improve homogenous catalysis in organic synthesis. ${ }^{33}$ In contrast, the field of electroanalytical sensors routinely use electrode functionalization to engender selectivity for a specific analyte, even in the presence of species with nearly identical reduction potentials on unmodified electrode surfaces. ${ }^{34,35}$ This selectivity arises from to the ability of the Ag-nanoparticles to lower the overpotential ${ }^{36}$ required to reduce or oxidize an analyte of interest. ${ }^{37}$ Additionally, selectivity can manifest as a change in the reduction potential (potential shift to less driving potentials of the voltametric wave) through metal particleanalyte interactions. ${ }^{38-42}$ Many analyte-specific sensors have been developed using Agnanoparticle decorated electrodes. ${ }^{38-41}$ Preparation of $\mathrm{Ag}$ modified electrodes for sensor applications includes drop casting a suspension of pre-formed Ag-nanoparticles onto a surface and drying, or by the cathodic reduction of a solution of silver(I), with the latter being strikingly similar to the procedure used in this developed cross coupling reaction. ${ }^{43}$ To better understand the interplay between the electrode surface modification by silver and homogenous nickel catalysis, several experiments were conducted.

First, modified electrode surfaces were characterized using Scanning Electron Microscopy (SEM) imaging, Transmission Electron Microscopy (TEM) and Energy-Dispersive X-ray Spectroscopy (EDS)(Figure 4B). When $\mathrm{AgNO}_{3}$ alone was electrodeposited prior to the start of the reaction, the glassy carbon electrodes were coated with a gray film. The use of these modified electrodes yielded only $24 \%$ of product 39 between RAE 7 and vinyl iodide 5. SEM imaging showed that while large silver crystals formed (1-5 $\mu \mathrm{m}$ in diameter), there were very low levels of nanoparticulate silver on the electrode surface. However, addition of $\mathrm{LiCl}$ to this pre-reaction electrodeposition produced electrodes with improved reaction performance (41\% NMR yield). SEM and TEM analyses of these electrode surfaces revealed the presence of nanoparticulate silver in sizes ranging 10-100 $\mathrm{nm}$ in diameter (See SI, Figures S36-40). Controls verified the need for a halide source but, $\mathrm{LiCl}$ was not required in the coupling reactions since $\mathrm{NiCl}_{2}$ could serve as the halide donor in the preparative reactions. The reaction of $\mathrm{AgNO}_{3}$ and halides in solution will produce photosensitive silver halide salts that readily decompose. Electrodes modified using a $\mathrm{AgNO}_{3}$ and $\mathrm{LiCl}$ solution that had been allowed to stir for several minutes before electrolysis were evaluated in the coupling reaction. In such cases, only a $17 \%$ yield of $\mathbf{3 9}$ was obtained and very little nanoparticulate silver was observed in microscopic characterization (See SI, Figure S44-45). Collectively, these results suggest that nanoparticulate silver electrodeposited before the crosscoupling reaction, is present and responsible, at least in part, for the improved performance.

Seeking to compare reactivity of these nanoparticle coated electrodes in the cross-coupling with known reactivity of similarly functionalized electrodes, glassy carbon disk electrodes were also functionalized. Nanoparticle deposition on this disk electrode was validated by anodic stripping voltammetry (Figure 4B) and S/TEM imaging (See SI, Figure S50-51). ${ }^{38}$ The reduction potential of benzyl chloride is known to shift $500 \mathrm{mV}$ more positive on a silver-nanoparticle decorated electrode and such behavior was observed on our nanoparticle-coated disk. ${ }^{41}$ Cyclic 
voltametric studies of reductively labile components of the cross coupling, $\mathrm{NiCl}_{2}$ (bpy) and $\mathrm{RAE}$ 7, revealed no significant differences in the onset of reduction of these two species at the functionalized electrode in comparison to glass carbon surfaces (See SI, Figures S54-76).

The kinetic behavior of the modified electrode was then studied using rotating disk electrode (RDE) voltammetry. ${ }^{44}$ In the case of $\mathrm{NiCl}_{2}$ (bpy), a diminished current response in consecutive cycles at an unmodified glassy carbon RDE is observed. When the same measurements were performed with the silver-nanoparticle modified RDE, the peak current response of the $\mathrm{Ni}(\mathrm{II}) / \mathrm{Ni}(0)$ redox couple was slightly lower and its current also decreased with continuous cycling, but at a notably slower rate (Figure 4C-I). The initial current response could be restored to the glassy carbon electrode by a potential excursion to $+1.5 \mathrm{~V}$ vs. $\mathrm{Ag} / \mathrm{AgCl}$, indicating that passivation is likely occurring through over-reduction and deposition of the catalyst on the electrode surface. Supporting this hypothesis, the cell potential (at constant current) of the reaction revealed a significant difference between the reactions with and without $\mathrm{AgNO}_{3}$. The potential of the reaction in the presence of $\mathrm{AgNO}_{3}\left(\mathrm{E}_{\text {cell }}=-1.15 \mathrm{~V}\right.$ vs. $\left.\mathrm{Ag} / \mathrm{AgCl}\right)$ was $510 \mathrm{mV}$ more positive than the reaction without silver $\left(\mathrm{E}_{\text {cell }}=-1.66 \mathrm{~V}\right.$ vs. $\left.\mathrm{Ag} / \mathrm{AgCl}\right)$. This shift in potential (effectively a lower overpotential) could prevent the over-reduction of the catalyst which we believe to be responsible for the passivation of the electrode. To test this hypothesis (Figure 4CII), the cross-coupling reaction was run at a constant potential of $-1.15 \mathrm{~V}$ vs. $\mathrm{Ag} / \mathrm{AgCl}$ without a silver salt. These conditions resulted in a $10 \%$ increase in the yield of $\mathbf{3 9}$. Furthermore, a reaction conducted with intermittent potential excursions to $+1.5 \mathrm{~V}(70 \mathrm{~s}$ at $+1.5 \mathrm{~V}$ vs. $\mathrm{Ag} / \mathrm{AgCl}$ for every 11.5 mins of $-6 \mathrm{~mA}$ ), resulted in an $11 \%$ increase in the yield of $\mathbf{3 9}$, consistent with the results of the RDE experiments and further providing evidence for catalyst over-reduction, and electrode passivation at more forcing potentials - a deleterious process partially obviated by the silver layer.

A comparison of the Levich (I vs $\omega^{-1 / 2}$ ) analysis (Figure 4C-III) of the catalyst and the RAE 7 showed that diffusion of $\mathrm{NiCl}_{2}$ (bpy) was not significantly impacted by the silver nanoparticle layer (the calculated diffusion coefficient decreased by a factor of 2). ${ }^{45}$ However, 7 showed a significant change in its diffusion behavior on the silver nanoparticle functionalized RDE. While at slow rates of rotation the currents at the bare and Ag modified electrodes are comparable, at faster rates of rotations there is a clear divergence. Moreover, upon extrapolation to zero rotation, the intercept is clearly non-zero, suggesting possible adsorptive effects. Taken collectively with the results of reaction progress over time (See SI, Figure S19-20), these results suggest that a decreased amount of direct reduction of RAE at the cathode is likely responsible, at least in part, for the improved reaction yield.

Investigations of the vinyl iodides 5 and $\mathbf{4 0}$ revealed that while direct reduction does not occur, significant differences in behavior exist between these two model vinyl iodides when $\mathrm{NiCl}_{2}$ (bpy) and RAE are present (Figure 4C-IV). When RDE voltammetry was conducted using $\mathrm{NiCl}_{2}$ (bpy) and 40, a catalytic current response was observed at the $\mathrm{Ni}(0) / \mathrm{Ni}(\mathrm{II})$ redox couple (resulting from the oxidative addition of the vinyl iodide) consistent with ECcat kinetics and the previously mentioned electrode passivation. Furthermore, in the presence of RAE 7, this passivation behavior disappeared when using the methyl ester vinyl iodide 40. Interestingly, use of halo-acid $\mathbf{5}$ in these measurements generated a catalytic current without significant electrode passivation even in the absence of RAE. Surprisingly, after addition of RAE $\mathbf{7}$ to halo-acid $\mathbf{5}$ and $\mathrm{NiCl}_{2}$ (bpy), the second reduction wave was still observed at $-1.48 \mathrm{~V}$ vs $\mathrm{Ag} / \mathrm{AgCl}$ potential. We propose this wave is the electrochemical reduction of a nickel (II/III) alkenyl to a nickel (I) alkenyl intermediate. ${ }^{46,47}$ This profound difference in behavior when using halo-acid $\mathbf{5}$ instead of methyl ester 40 has significant implications on the detrimental behavior of carboxylic acids on reaction 
yield in the absence of $\mathrm{AgNO}_{3}$. Recent evidence has emerged suggesting the deactivation of nickel (I) intermediates through dimerization or aggregation. ${ }^{48}$ One can also imagine how this reduced species could accelerate the consumption of RAE in the homogeneous environment. ${ }^{49}$ Comparing the onset of these two reduction waves to the cell potential of the reaction without $\mathrm{AgNO}_{3}\left(\mathrm{E}_{\text {cell }}=\right.$ $-1.66 \mathrm{~V}$ vs. $\mathrm{Ag} / \mathrm{AgCl})$ and with $\mathrm{AgNO}_{3}\left(\mathrm{E}_{\text {cell }}=-1.15 \mathrm{~V}\right.$ vs. $\left.\mathrm{Ag} / \mathrm{AgCl}\right)$ is particularly informative. This finding implied that the Ag-NP layer decreases overpotential to the point immediately prior to the second reduction wave whereas with the use of bare glassy carbon electrodes, more reducing potentials beyond the second wave under standard reaction conditions (constant current) are applied.

In summary, it appears that the silver-nanoparticle layer on the electrode has several effects on the reaction components. First, the silver-NP layer lowers the overpotential preventing catalyst over-reduction and electrode passivation. Second, the lower overpotential also reduces the formation of $\mathrm{Ni}(\mathrm{I})$-alkenyl intermediates which appear to form even in the presence of RAEs when using vinyl iodides bearing carboxylic acids. Third, this layer slows mass transport and reduction of RAEs at the electrode surface likely from complications arising with adsorptive behavior, while diffusion of the catalyst is only minorly affected. While no singular result explains the role of the silver-nanoparticle layer, we hypothesize that the overall benefit observed is likely the result of the findings discussed above working together in concert. Further interdisciplinary studies between synthetic, electroanalytical, and materials specialists will likely provide deeper insights into this unique discovery.

This study of terpene synthesis has provided an efficient platform for the modular construction of polyunsaturated molecules with precise geometrical control. Its implementation required methodological advancement thereby revealing a relationship between productive catalysis and materials science. Electrochemistry offers chemists new enabling opportunities through variables that are uniquely available to it. While electrode modification offers new possibilities for synthetic chemists to optimize difficult reactions-analytical chemists and physical chemists have long embraced this concept to overcome their own chemical challenges. The potential of these unique parameters to enable useful chemical reactivity is an attractive area for further study.

Acknowledgments: We thank D.-H. H. and L. P. for assistance with NMR spectroscopy; J. Chen, B Sanchez, and E. Sturgell for (Scripps Automated Synthesis Facility) for assistance with HPLC, HRMS, and LCMS; A. Rheingold, M. Gembicky, and E. Samolova, (University of California, San Diego) for assistance with x-ray crystallography; we are grateful to University of Utah shared facilities of the Micron Technology Foundation Inc.; We thank J. Vantourout, Kawamata, Y., S. Gnaim., Y.Y. See, J. Edwards, K. McClymont, C. Bi and B. Smith for insightful discussions; and K. Eberle, G. Laudadio, A. Garrido-Castro, and M. Condakes for assistance in the preparation of the manuscript.

Funding: Financial support for this work was provided by the NIH (MIRA GM-118176), NSF Center for Synthetic Organic Electrochemistry (CHE-2002158) and the Microscopy Suite sponsored by the College of Engineering, Health Sciences Center, Office of the Vice President for Research, the Utah Science Technology and Research (USTAR) initiative of the State of Utah and, in part, by the MRSEC Program of the NSF under Award No. DMR-1121252. S.J.H. 
thanks the Fletcher-Jones Foundation for fellowship funding. M.D.P. thanks Richard and Nicola Lerner for the Endowed Fellowship.

\section{References}

1. Nicolaou, K. C.; Sorenson, E. J., Classics in Total Synthesis: Targets, Strategies, Methods. 1997; p xxii - 798.

2. Cox-Georgian, D.; Ramadoss, N.; Dona, C.; Basu, C., Therapeutic and Medicinal Uses of Terpenes. Medicinal Plants 2019, 333 - 359.

3. Jansen, D. J.; Shenvi, R. A., Synthesis of medicinally relevant terpenes: reducing the cost and time of drug discovery. Future Med. Chem. 2014, 6 (10), 1127-1148.

4. Serra, S., Chapter 7 - Recent Developments in the Synthesis of the Flavors and Fragrances of Terpenoid Origin. In Studies in Natural Products Chemistry, Atta ur, R., Ed. Elsevier: 2015; Vol. 46, pp 201-226.

5. Johnson, W. S.; Gravestock, M. B.; McCarry, B. E., Acetylenic bond participation in biogenetic-like olefinic cyclizations. II. Synthesis of dl-progesterone. J. Am. Chem. Soc. 1971, 93 (17), 4332-4334.

6. Yoder, R. A.; Johnston, J. N., A Case Study in Biomimetic Total Synthesis: Polyolefin Carbocyclizations to Terpenes and Steroids. Chem. Rev. 2005, 105 (12), 4730-4756.

7. Ungarean, C. N.; Southgate, E. H.; Sarlah, D., Enantioselective polyene cyclizations. Org. Biomol. Chem. 2016, 14 (24), 5454-5467.

8. Thirsk, C.; Whiting, A., Polyene natural products. J. Chen. Soc. Perkin Trans. I 2002, (8), 999-1023.

9. Negishi, E.-i.; Wang, G.; Rao, H.; Xu, Z., Alkyne Elementometalation-Pd-Catalyzed Cross-Coupling. Toward Synthesis of All Conceivable Types of Acyclic Alkenes in High Yields, Efficiently, Selectively, Economically, and Safely: "Green” Way. J. Org. Chem. 2010, 75 (10), 3151-3182.

10. Li, J.; Grillo, A. S.; Burke, M. D., From Synthesis to Function via Iterative Assembly of N-Methyliminodiacetic Acid Boronate Building Blocks. Acc. Chem. Res. 2015, 48 (8), $2297-$ 2307.

11. Dewick, P. M., The mevalonate and methylerythritol phosphate pathways: Terpenoids and steroids. Medicinal Natural Products; A biosynthetic approach 2002, 2, 167-289.

12. Smith, J. M.; Harwood, S. J.; Baran, P. S., Radical Retrosynthesis. Acc. Chem. Res. 2018, 51 (8), 1807-1817.

13. Gaich, T.; Baran, P. S. Aiming for the Ideal Synthesis. J. Org. Chem. 2010, 75, 46574673.

14. Edwards, J. T.; Merchant, R. R.; McClymont, K. S.; Knouse, K. W.; Qin, T.; Malins, L. R.; Vokits, B.; Shaw, S. A.; Bao, D.-H.; Wei, F.-L.; Zhou, T.; Eastgate, M. D.; Baran, P. S., Decarboxylative alkenylation. Nature 2017, 545 (7653), 213-218.

15. Cannes, C.; Condon, S.; Durandetti, M.; Périchon, J.; Nédélec, J. Y., Nickel-Catalyzed Electrochemical Couplings of Vinyl Halides: Synthetic and Stereochemical Aspects. J. Org. Chem. 2000, 65 (15), 4575-4583.

16. Novaes, L. F. T.; Liu, J.; Shen, Y.; Lu, L.; Meinhardt, J. M.; Lin, S., Electrocatalysis as an enabling technology for organic synthesis. Chem. Soc. Rev. 2021, 50 (14), 7941-8002. 
17. Campelo, J. M.; Luna, D.; Luque, R.; Marinas, J. M.; Romero, A. A., Sustainable Preparation of Supported Metal Nanoparticles and Their Applications in Catalysis.

ChemSusChem 2009, 2 (1), 18-45.

18. Perkins, R. J.; Pedro, D. J.; Hansen, E. C., Electrochemical Nickel Catalysis for Sp2-Sp3 Cross-Electrophile Coupling Reactions of Unactivated Alkyl Halides. Org. Lett. 2017, 19 (14), 3755-3758.

19. Slegeris, R.; Dudley, G. B., Alternative synthetic approaches to rac-progesterone by way of the classic Johnson cationic polycyclization strategy. Tetrahedron 2016, 72 (26), 3666-3672.

20. Camelio, A. M.; Johnson, T. C.; Siegel, D., Total Synthesis of Celastrol, Development of a Platform to Access Celastroid Natural Products. J. Am. Chem. Soc. 2015, 137 (37), 1186411867.

21. Snider, B. B.; Kiselgof, J. Y.; Foxman, B. M., Total Syntheses of ( \pm )-Isosteviol and ( \pm )Beyer-15-ene-3 $\beta, 19$-diol by Manganese(III)-Based Oxidative Quadruple Free-Radical Cyclization. J. Org. Chem. 1998, 63 (22), 7945-7952.

22. Walker, R. P.; Faulkner, D. J., Diterpenes from the sponge Dysidea amblia. J. Org. Chem. 1981, 46 (6), 1098-1102.

23. Thompson, J. E.; Walker, R. P.; Faulkner, D. J., Screening and bioassays for biologically-active substances from forty marine sponge species from San Diego, California, USA. Mar. Biol. 1985, 88 (1), 11-21.

24. Serra, S.; Lissoni, V., First Enantioselective Synthesis of Marine Diterpene Ambliol-A. Eur. J. Org. Chem. 2015, 2015 (10), 2226-2234.

25. Scheffrahn, R. H.; Gaston, L. K.; Sims, J. J.; Rust, M. K., Identification of the defensive secretion from soldiers of the North American termite,Amitermes wheeleri (Desneux) (Isoptera: Termitidae). J. Chem. Ecol. 1983, 9 (9), 1293-1305.

26. Roelofs, W. L.; Gieselmann, M. J.; CardÉ, A. M.; Tashiro, H.; Moreno, D. S.; Henrick, C. A.; Anderson, R. J., Sex pheromone of the California red scale, Aonidiella aurantii. Nature 1977, 267 (5613), 698-699.

27. Weix, D. J., Methods and Mechanisms for Cross-Electrophile Coupling of Csp2 Halides with Alkyl Electrophiles. Acc. Chem. Res. 2015, 48 (6), 1767-1775.

28. Brito, C.; Jordão, V. L.; Pierce, G. J., Ambergris as an overlooked historical marine resource: its biology and role as a global economic commodity. J. Mar. Biol. Assoc. U. K. 2016, 96 (3), 585-596.

29. Barrero, A. F.; Altarejos, J.; Alvarez-Manzaneda, E. J.; Ramos, J. M.; Salido, S., Synthesis of ( \pm -Ambrox from (E)-Nerolidol and $\beta$-Ionone via Allylic Alcohol [2,3] Sigmatropic Rearrangement. J. Org. Chem. 1996, 61 (6), 2215-2218.

30. Schubert, V.; Dietrich, A.; Ulrich, T.; Mosandl, A., The Stereoisomers of Nerolidol: Separation, Analysis and Olfactoric Properties. Zeitschrift für Naturforschung C 1992, 47 (3-4), 304-307.

31. Ben Salha, G.; Abderrabba, M.; Labidi, J., A status review of terpenes and their separation methods. Rev. Chem. Eng. 2021, 37 (3), 433-447.

32. Chan, W.-K.; Tan, L. T.; Chan, K.-G.; Lee, L.-H.; Goh, B.-H., Nerolidol: A Sesquiterpene Alcohol with Multi-Faceted Pharmacological and Biological Activities. Molecules 2016, 21 (5), 529.

33. Dong, X.-Y.; Gao, Z.-W.; Yang, K.-F.; Zhang, W.-Q.; Xu, L.-W., Nanosilver as a new generation of silver catalysts in organic transformations for efficient synthesis of fine chemicals. Catalysis Science \& Technology 2015, 5 (5), 2554-2574. 
34. Chillawar, R. R.; Tadi, K. K.; Motghare, R. V., Voltammetric techniques at chemically modified electrodes. Journal of Analytical Chemistry 2015, 70 (4), 399-418.

35. Zen, J.-M.; Senthil Kumar, A.; Tsai, D.-M., Recent Updates of Chemically Modified Electrodes in Analytical Chemistry. Electroanalysis 2003, 15 (13), 1073-1087.

36. Nutting, J. E.; Gerken, J. B.; Stamoulis, A. G.; Bruns, D. L.; Stahl, S. S., "How Should I Think about Voltage? What Is Overpotential?": Establishing an Organic Chemistry Intuition for Electrochemistry. J. Org. Chem. 2021.

37. Luo, X.; Morrin, A.; Killard, A. J.; Smyth, M. R., Application of Nanoparticles in Electrochemical Sensors and Biosensors. Electroanalysis 2006, 18 (4), 319-326.

38. Welch, C. M.; Banks, C. E.; Simm, A. O.; Compton, R. G., Silver nanoparticle assemblies supported on glassy-carbon electrodes for the electro-analytical detection of hydrogen peroxide. Analytical and Bioanalytical Chemistry 2005, 382 (1), 12-21.

39. Fox, C. M.; Breslin, C. B., Electrochemical formation of silver nanoparticles and their applications in the reduction and detection of nitrates at neutral $\mathrm{pH}$. Journal of Applied Electrochemistry 2020, 50 (1), 125-138.

40. Karuppiah, C.; Muthupandi, K.; Chen, S.-M.; Ali, M. A.; Palanisamy, S.; Rajan, A.; Prakash, P.; Al-Hemaid, F. M. A.; Lou, B.-S., Green synthesized silver nanoparticles decorated on reduced graphene oxide for enhanced electrochemical sensing of nitrobenzene in waste water samples. RSC Adv. 2015, 5 (39), 31139-31146.

41. Isse, A. A.; Gottardello, S.; Maccato, C.; Gennaro, A., Silver nanoparticles deposited on glassy carbon. Electrocatalytic activity for reduction of benzyl chloride. Electrochemistry Communications 2006, 8 (11), 1707-1712.

42. Ren, X.; Meng, X.; Chen, D.; Tang, F.; Jiao, J., Using silver nanoparticle to enhance current response of biosensor. Biosens. Bioelectron. 2005, 21 (3), 433-437.

43. Manohar, A. B.; Bhalchandra, M. B., Silver Nanoparticles: Synthesis, Characterization and their Application as a Sustainable Catalyst for Organic Transformations. Current Organic Chemistry 2015, 19 (8), 708-727.

44. Compton, R. G.; Laing, M. E.; Mason, D.; Northing, R. J.; Unwin, P. R.; Rowlinson, J. S., Rotating disc electrodes: the theory of chronoamperometry and its use in mechanistic investigations. Proc. Math. Phys. Eng. Sci. 1988, 418 (1854), 113-154.

45. Levich, V.G., Phisiochemical Hydrodynamics, Prentice Hall, Englewood Cliffs, N.J. (1962).

46. C. Cannes, E. Labbé, M. Durandetti, M. Devaud, J.-Y. Nédélec. Nickel-catalyzed Electrochemical Homocoupling of Alkenyl Halides: Rates and Mechanisms. J. Electroanal. Chem. 1996, 412, 85-93.

47. Amatore, C.; Jutand, A.; Motteir, L. Mechanisms of Nickel-catalyzed Electron Transfer Activation of Aromatic Halides. Part 1: Biphenyl Electrosynthesis from Bromobenzene. J. Electroanal. Chem. 1991, 306, 125-140.

48. N. A. Till, S. Oh, D. W. C. MacMillan, M. J. Bird. The Application of Pulse Radiolysis to the Study of $\mathrm{Ni}(\mathrm{I})$ Intermediates in Ni-catalyzed Cross-Coupling Reactions. J. Am. Chem. Soc. 2021, 143, 9332-9337.

49. J. Cornella, J. T. Edwards, T. Qin, S. Kawamura, J. Wang, C.-M Pan, R. Gianatassio, M. Schmidt, M. D. Eastgate, P. S. Baran. Practical Ni-catalyzed Aryl-Alkyl Cross-Coupling of Secondary Redox-Active Esters. J. Am. Chem. Soc. 2016, 138, 2174-2177.

TOC 


\section{Modular Terpene Synthesis Enabled by Mild Electrocatalytic Couplings}

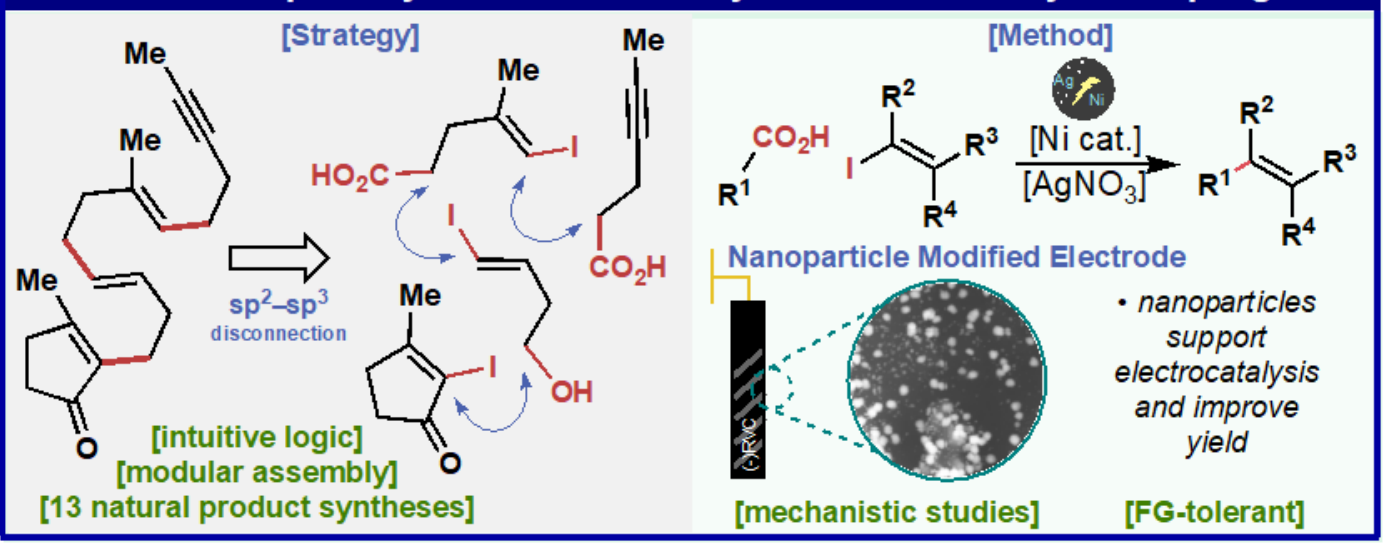

